INPLASY

PROTOCOL

To cite: Zhang et al.

Therapeutic effects of exercises for patients with chronic kidney disease: an overview of meta-analyses of clinical trials. Inplasy protocol 202070049. doi:

10.37766/inplasy2020.7.0049

Received: 13 July 2020

Published: 13 July 2020

Corresponding author:

Fan Zhang

1051071914@qq.com

Author Affiliation:

Longhua Hospital Shanghai

University of Traditional

Chinese Medicine

Support: There is no funding.

Review Stage at time of this submission: The review has not yet started.

Conflicts of interest:

There is no conflict of interest.

\section{Therapeutic effects of exercises for patients with chronic kidney disease: an overview of meta-analyses of clinical trials}

Zhang, $\mathrm{F}^{1}$; Bai, $\mathrm{Y}^{2}$; Zhao, $\mathrm{X}^{3}$; Zhang, $\mathrm{Y}^{4}$; Huang, LY5; Wang, $\mathrm{H}^{6}$; Zhang, $\mathrm{HC}^{7}$.

Review question / Objective: The aim of this overview was to systematically appraise and summarize meta-analyses investigating the effect of exercises compared with a control condition on cardiovascular risk factor, physical fitness, health-related quality of life, dialysis-related symptoms in patients with CKD.

Condition being studied: Exercise is a proven therapy that is associated with numerous health benefits in patients with CKD, and CKD patients are now encouraged to participate in sufficient levels of regular exercise in daily life. A growing systematic review and meta-analyses has examined the role of exercise in treatment of CKD. Where multiple metaanalyses have been published on similar and overlapping questions over a relatively short timeframe, there is a need for an overview of the meta-analysis data, namely umbrella review, to determine the overarching quality and strength of the evidence and the level of consistency or potential contradiction in outcomes.

INPLASY registration number: This protocol was registered with the International Platform of Registered Systematic Review and Meta-Analysis Protocols (INPLASY) on 13 July 2020 and was last updated on 13 July 2020 (registration number INPLASY202070049).

\section{INTRODUCTION}

Review question / Objective: The aim of this overview was to systematically appraise and summarize meta-analyses investigating the effect of exercises compared with a control condition on cardiovascular risk factor, physical fitness, health-related quality of life, dialysisrelated symptoms in patients with CKD.

Rationale: Chronic kidney disease (CKD) represents a large burden of morbidity across the global affecting about 10.4\% 16 $\%$ of all adults. In recent years, with the 
rising incidence of hypertension, and diabetes mellitus, the number of patients with CKD will still gradually increase. This growing patients with CKD experiences ongoing physical and psychological side effects associated with their disease or treatment and is more likely to suffer from another chronic disease.

Condition being studied: Exercise is a proven therapy that is associated with numerous health benefits in patients with CKD, and CKD patients are now encouraged to participate in sufficient levels of regular exercise in daily life. A growing systematic review and metaanalyses has examined the role of exercise in treatment of CKD. Where multiple metaanalyses have been published on similar and overlapping questions over a relatively short timeframe, there is a need for an overview of the meta-analysis data, namely umbrella review, to determine the overarching quality and strength of the evidence and the level of consistency or potential contradiction in outcomes.

\section{METHODS}

Search strategy: A systematic search was conducted using the following database: PubMed, the Cochrane Library, Web of Science, Embase. In addition, a manual review of references from similar systematic review articles was screened to trace additional relevant studies. The search phrase consisted of MeSH terms and keywords related to exercise, metaanalysis and chronic kidney disease.

Participant or population: Patients diagnosed chronic kidney disease whether it's dialysis or not.

Intervention: Exercise, including aerobic exercise, resistance training, aerobic and resistance training combined, or other exercise-based therapy.

Comparator: Sham/no exercise or usual/ standard care.
Study designs to be included: Systematic reviews with meta-analysis of RCTs and quasi-experimental studies.

Eligibility criteria: Eligibility criteria were required to (1) include patients diagnosed CKD whether it's dialysis or not; (2) compare exercise therapy no matter types with sham/no exercise or usual/standard care; (3) outcomes were based on cardiovascular risk factor, physical fitness, health-related quality of life, dialysisrelated symptoms; (4) be systematic review with meta-analysis of controlled parallel intervention trials (ie, systematic reviews with meta-analysis of RCTs and quasiexperimental studies were eligible, systematic reviews with meta-analysis of observational prospective studies were excluded).

Information sources: A systematic search was conducted using the following database: PubMed, the Cochrane Library, Web of Science, Embase. In addition, a manual review of references from similar systematic review articles was screened to trace additional relevant studies. The search phrase consisted of MeSH terms and keywords related to exercise, metaanalysis and chronic kidney disease. Article language was restricted in English.

Main outcome(s): Cardiovascular risk factor.

Additional outcome(s): Physical fitness, health-related quality of life, dialysisrelated symptoms.

Quality assessment / Risk of bias analysis: The risk of bias of each included metaanalysis will be evaluated using the AMSTAR checklist. This tool contains 11 short, concrete questions, and each question has response options: yes, no, can't answer and not applicable. Q1 = Was an "a priori" design provided? Q2 = Was there duplicate study selection and data extraction? Q3 = Was a comprehensive literature search perform? Q4 = Was the status of publication (i.e. grey literature) used as an inclusion criterion? Q5 = Was a list of studies (included and excluded) 
provided? Q6 $=$ Were the characteristics of the included studies provided? Q7 = Was the scientific quality of the included studies assessed and documented? Q8 = Was the scientific quality of the included studies used appropriately in formulation conclusions? Q9 = Were the methods used to combine the findings of studies appropriate? Q10 = Was the likelihood of publication bias assessed? Q11 = Was the conflict of interest included?

Strategy of data synthesis: The metaanalyses were classified according to exercise type into four categories: (1) aerobic exercise, (2) resistance training, (3) aerobic and resistance training combined, (4) other exercise-based therapy. For each category, the data were analyses qualitatively based on the SMDs of each included outcome. If there were not presented as SMD in the original metaanalysis, the software of Review Manager 5.3 was used to convert the outcomes to SMD to allow visual comparison of the results in a forest plot. If data were insufficient for conversion, data were extracted from original RCTs and processed as instructed by the Cochrane Collaboration. Effects are considered, small $(<0.5)$, moderate $(0.50-0.79)$ and large $(\geq 0.80)$. The Grading of Recommendations Assessment, Development and Evaluation (GRADE) system was used to evaluate the quality of evidence provided by each individual meta-analysis.16 Quality of evidence was considered 'high' (further research is very unlikely to change our confidence in the effect estimated by this meta-analysis), 'moderate' (further research is likely to have an important impact on our confidence in the effect estimated by this meta-analysis and may change the estimate), 'low' (further research is very likely to have an important impact on our confidence in the effect estimated by this meta-analysis and is likely to change the estimate) and 'very low' (any effect estimated by this metaanalysis is very uncertain).

Subgroup analysis: The meta-analyses were classified according to exercise type into four categories: (1) aerobic exercise,
(2) resistance training, (3) aerobic and resistance training combined, (4) other exercise-based therapy.

Sensibility analysis: Not applicable.

Language: English.

Country(ies) involved: China.

Keywords: Exercise; Chronic kidney disease; Systematic review; Meta-analysis.

Contributions of each author:

Author 1 - Fan Zhang - Author 1 will develop the search strategy and draft the manuscript.

Author 2 - Yan Bai - Author 2 will independently work on study selection, quality assessment and data extraction.

Author 3 - Xing Zhao - Author 3 will independently work on study selection, quality assessment and data extraction.

Author 4 - Ying Zhang - Author 4 will work on data synthesis.

Author 5 - Liuyan Huang - Author 5 will assess the full-text against the inclusion criteria.

Author 6 - Hui Wang - Author 6 will assess the full-text against the inclusion criteria.

Author 7 - Huachun Zhang - Author 7 will resolve any divergences. 